\title{
Pratiques
}

Linguistique, littérature, didactique

\section{Théories linguistiques et littéraires et ateliers d'écriture}

\section{Michèle Monte}

\section{(2) OpenEdition}

Journals

\section{Édition électronique}

URL : http://journals.openedition.org/pratiques/3515

DOI : $10.4000 /$ pratiques.3515

ISSN : 2425-2042

\section{Éditeur}

Centre de recherche sur les médiations (CREM)

\section{Édition imprimée}

Date de publication : 15 décembre 2012

Pagination : 205-222

\section{Référence électronique}

Michèle Monte, «Théories linguistiques et littéraires et ateliers d'écriture », Pratiques [En ligne], 155-156 | 2012, mis en ligne le 18 décembre 2017, consulté le 19 avril 2019. URL : http:// journals.openedition.org/pratiques/3515; DOI : 10.4000/pratiques.3515

(c) Tous droits réservés 


\title{
Théories linguistiques et littéraires et ateliers d'écriture
}

\author{
Michèle Monte
}

Université du Sud-Toulon-Var, BABEL EA 2649

\begin{abstract}
L'apparition des ateliers d'écriture dans les années $1970^{(1)}$ doit beaucoup au renouvellement des théories littéraires et linguistiques dans les années 1955-1970. Ces années, on le sait, furent marquées à la fois par une forte effervescence théorique et par d'intenses débats idéologiques et politiques nourris par la guerre froide, l'opposition entre le bloc capitaliste et le bloc communiste, les guerres de décolonisation puis le mouvement hippie et les révoltes étudiantes. Deux revues, Tel Quel (19601982) et Change (1968-1983), qui ont alimenté les réflexions et les pratiques des créateurs des ateliers d'écriture, ont activement contribué à ce bouillonnement d'idées. À les lire, on est frappé à la fois par la richesse des analyses théoriques, dues notamment aux animateurs du Nouveau Roman et à leurs successeurs, par l'audace des explorations formelles, et par le large accueil qui est fait aux théories linguistiques de Noam Chomsky, d'une part, des formalistes russes et du cercle de Prague d'autre part. Jean Ricardou et Jean-Pierre Faye incarnent cette symbiose entre réflexion théorique et pratique scripturale : le premier, qui joua un rôle décisif à l'origine du courant des ateliers d'écriture représenté par Claudette Oriol-Boyer, publie quatorze fois dans Tel Quel de 1960 à 1969 et le second, qu' Anne Roche évoque comme une de ses sources d'inspiration, après avoir publié dans Tel Quel et s'être brouillé avec le collectif dirigé par Sollers, anime la revue Change dans laquelle il écrit notamment «Théorie du récit » $\left(n^{\circ} 5\right)$, « Le mouvement du change des formes » $\left(n^{\circ} 18\right)$, « Narration nouvelle et transformationnelle » $\left(n^{\circ} 34 / 35\right)$.
\end{abstract}

Je voudrais montrer tout d'abord dans cet article ce que les ateliers d'écriture doivent à ce climat intellectuel, tout en évoquant brièvement les divergences entre les différents courants d'ateliers d'écriture. Dans une deuxième partie, je m'intéresserai à la situation actuelle des ateliers d'écriture, en examinant quelques-unes de leurs

(1) Sur l'émergence des ateliers d'écriture et les différents courants qui s'y manifestent, on lira Boniface, C. 1992 et Rossignol, I., 1996. 
évolutions, et en interrogeant leurs rapports avec l'institution scolaire d'une part, avec les théories littéraires et linguistiques récentes, d'autre part ${ }^{(2)}$.

\section{Les années 60-80 : un ancrage théorique fort au service d'une nouvelle vision de l'écriture}

\subsection{Contre la littérature représentative, l'imitation et le pillage intertexuel}

Malgré les résistances de l'Université ${ }^{(3)}$, la théorie littéraire se modifie fortement à partir des années 1955 en raison de la prépondérance qu'acquièrent dans le champ scientifique le structuralisme et la linguistique de Jakobson et Chomsky. Les nouvelles théories du texte remettent tout d'abord fortement en cause le principe hérité d'Aristote d'une littérature chargée de représenter le monde. Ce sont Jean Ricardou et les romanciers du Nouveau Roman, Robbe-Grillet surtout, qui ont le plus insisté sur cette critique de la littérature comme mimésis, lui opposant la construction du monde par le texte lui-même. Mais on peut aussi citer ce passage d'un entretien de Barthes à Tel Quel en 1961 :

L'œuvre la plus « réaliste » ne sera pas celle qui «peint » la réalité, mais qui, se servant du monde comme contenu (ce contenu lui-même est d'ailleurs étranger à sa structure, c'est-à-dire à son être), explorera le plus profondément possible la réalité irréelle du langage. (Tel Quel $\mathrm{n}^{\circ}$ 7, 1961, p. 40)

La position de Barthes dans cet entretien est beaucoup plus nuancée que la citation ne pourrait le laisser croire, car il montre bien aussi les liens qui unissent la littérature au monde, mais, dans cette époque d'intense conflit théorique, tout ce qui peut permettre de rompre avec ce que Ricardou appelle le « dogme de l'expression » est bon à prendre. C'est pourquoi la fameuse fonction poétique de Jakobson, caractérisée par « la visée (Einstellung) du message en tant que tel » recevra un franc succès : en effet, pour le linguiste, « cette fonction, qui met en évidence le côté palpable des signes, approfondit par là même la dichotomie fondamentale des signes et des objets » (1963 : 218). Faisant de cette fonction "la fonction dominante, déterminante, de l'art du langage », Jakobson permet de relativiser les autres fonctions - référentielle, conative, émotive - dans le texte littéraire et autorise certains à ériger la littérature en pratique autotélique, excluant toute référence au réel.

Or un tel renversement était capital pour les ateliers d'écriture car il permettait de déplacer l'accent du quelque chose à dire, préalable à l'écriture, vers le travail de l'écriture elle-même. Il s'agissait de libérer les scripteurs de l'angoisse du « je n'ai rien à dire » et de les faire évoluer d'une vision de l'écriture comme déversoir d'émotions vers un travail plus réfléchi de mise à distance. C'est pourquoi la célèbre phrase d'Aragon dans Je n'ai jamais appris à écrire ou les incipit - « Je crois encore qu'on pense à partir de ce qu' on écrit, et pas le contraire»-est souvent évoquée ${ }^{(4)}$, et

(2) Je reprendrai l'étude des liens entre théories littéraires et linguistiques et ateliers d'écriture du point de vue des inventeurs d'ateliers dans ma contribution aux actes du colloque de Cerisy 2011, Ateliers d'écriture littéraire, organisé par Cl. Oriol-Boyer et D. Bilous, à paraitre en 2014.

(3) Pour ne citer qu'un exemple, une levée de boucliers accueillit la parution de l'étude sur «Les Chats » de Baudelaire écrite à quatre mains par Jakobson et Lévi-Strauss.

(4) Citons, entre autres, André (1989) dès l'introduction (p. 10) et Neumayer (2003) en exergue à la présentation de l'atelier 3, dans la partie «L'entrée en écriture » (p. 48). 
que l'une de ses nouvelles «Le mentir-vrai » est fréquemment utilisée pour inventer des consignes d'atelier. Cette conviction s'allie à la pratique du détournement de texte, légitimée par l'Oulipo, et se fonde sur la théorisation de l'intertextualité, - le terme est popularisé par Kristeva (1969) - facilitée par la diffusion des travaux du cercle de Bakhtine à partir de 1970.

Du côté des ateliers d'écriture, le «bref préambule théorique » de Roche, Guiguet et Voltz (1989) s'ouvre précisément par une partie intitulée «Dialogue de textes ou l'intertextualité » qui veut libérer les scripteurs du souci de l'originalité. On retrouvera cette posture dans la plupart des livres consacrés aux ateliers d'écriture, par exemple dans le livre de Françoise Weck :

Le travail d'écriture s'opérant à partir d'un déjà-écrit, l'hypothèque d'un quelque chose à dire est levée et la prise en compte du matériau langagier en est facilitée : l'obsession thématique écartée, il devient possible d'élaborer librement, à partir du réservoir langagier que constitue le texte, des significations neuves dégagées d'un vouloir dire préexistant. (p. 36)

Les parodies, qui font l'objet de plusieurs articles dans Change et expliquent notamment l'intérêt de cette revue pour Rabelais, et les autres formes de réécriture, étudiées systématiquement dans Palimpsestes de Genette ainsi que dans les travaux de Daniel Bilous $(2008,2009)$ sur la « littérature au second degré », sont des éléments souvent présents dans les ateliers d'écriture, notamment autour du conte de fées ${ }^{(5)}$.

\subsection{Contre le génie singulier, les contraintes formelles et le travail collectif}

La sémantique structurale de Greimas et la narratologie en plein essor vont aussi irriguer les ateliers d'écriture en donnant aux participants des outils simples pour construire des récits longs. Je ne développe pas ces points qui sont bien connus, préférant relever l'intérêt de Jean-Pierre Faye à la fois pour Émile Benveniste et pour Chomsky, ce qui peut paraître antinomique. Dans les articles qu'il consacre à la théorie du récit, Faye, qui reprend la distinction entre " procès de l'énoncé » et « procès de l'énonciation » (Change 5, 1970, p. 8) et se réfère à l'article de Benveniste sur « Les relations de temps dans le verbe français ${ }^{(6)}$, insiste sur la double temporalité du récit, qui énonce une action passée mais tout aussi bien « la produit» (p. 10). Le structuralisme n'est pas mis au service d'une vision anhistorique de la littérature mais au contraire permet de penser de façon économique le passage d'une forme à une autre. La revue fait une large part aux formalistes russes et à Chomsky parce qu'elle est centrée sur les « changes de formes » : il s'agit de produire de nouveaux récits à partir de structures en nombre relativement limité, de même que les structures syntaxiques permettent d'engendrer un nombre infini de phrases à partir d'un petit nombre de règles de réécriture. La réflexion sur les formes passe aussi par une réévaluation de l'héritage littéraire qui privilégie les « formalistes » sur les adeptes de l'inspiration. Cette tendance est très nette dans Change, qui ouvre largement ses pages à Jacques Roubaud. Celui-ci s'emploie à définir «ce que la littérature dit du langage » (Change 6, 1970, p. 11) en insistant sur la littérature comme «mémoire, et code, d'une langue, et du

(5) On peut citer, pour la revue Pratiques, le n ${ }^{\circ} 42 L^{\prime}$ écriture-imitation et notamment les articles de Petitjean (1984a et b).

(6) Paru en 1959 et repris dans Problèmes de linguistique générale, Gallimard 1966. 
langage » (p. 12). Il consacre plusieurs articles à la métrique, mais son intérêt pour les formes fixes n'implique aucun passéisme : Roubaud définit précisément le vers libre comme une contrainte nouvelle, spécifique, de la poésie contemporaine. Par son attention à première vue paradoxale pour les littératures du passé ${ }^{(7)}$, Change propose une réflexion stimulante sur les changements formels, et leurs interactions avec les changements sociaux.

Mais ce que les animateurs d'ateliers retiendront surtout, c'est la notion de contrainte d'écriture, en s'appuyant sur la théorie et la pratique de l'Oulipo, auquel appartient Roubaud ${ }^{(8)}$. Roche, Guiguet, Voltz affirment d'emblée dans leur préambule : " la contrainte vous porte, elle vous permet d'écrire alors même que vous pensiez "n'avoir rien à dire" » (p. 7) et elles consacrent leurs deux premiers chapitres à des exercices oulipiens. Par ailleurs les références à Perec et Calvino sont légion dans les différents livres écrits par des animateurs d'ateliers d'écriture qui puisent dans leurs œuvres maintes consignes. La posture auctoriale (Bisenius-Penin 2005 : 213) de l'Oulipo convient aussi très bien aux animateurs d'ateliers : non seulement ses membres « réfutent le don, l'inspiration au profit de la notion d'artisanat» (ibid.) mais ils se conçoivent comme un auteur collectif et promeuvent une coopération, un échange des brouillons, qui vont être largement pratiqués dans les ateliers.

On sait que l'Oulipo pratique le centon qui consiste à produire un texte nouveau à partir de différents fragments empruntés à des auteurs différents. Cet intérêt pour le collage et le montage, souvent importés du cinéma ou des arts plastiques ${ }^{(9)}$, est assez général dans ces années 60-70. Outre qu'il s'oppose à la vision organiciste de l'œuvre unique et achevée, il apporte des outils et une légitimité aux animateurs d'ateliers soucieux de mettre en cohérence a posteriori des fragments produits indépendamment les uns les autres. Si certains ateliers, notamment ceux de l'Aleph, se proposent de guider les scripteurs vers la production de formes longues, la plupart en effet font produire des textes courts qui peuvent éventuellement être ensuite reliés les uns aux autres par des consignes. Le groupe d'Aix-en-Provence propose dans un sous-chapitre intitulé « Meccano » diverses possibilités de production de récits longs à partir de schémas préconstruits. O. et $\mathrm{M}$. Neumayer $\left(2003^{(10)}\right)$ consacrent toute leur troisième partie à la notion de dispositif permettant d'accueillir des fragments de genres et d'auteurs différents et réfléchissent également à la question de l'hypertexte.

Le terme de productions pour dénommer les textes apparait à ce moment-là dans un contexte clairement politique. Juste après les évènements de 1968, la référence à l'écriture comme travail non seulement s'oppose à l'idée d'inspiration, mais elle permet de soutenir une vision politique qui cherche à rapprocher travail intellectuel et travail manuel - le terme d'atelier s'inscrit dans ce paradigme - Le collectif Change définit le récit comme un travail de production qui, à partir de formes partiellement nouvelles et partiellement héritées, transforme une matière. On reprend volontiers le mot de Lautréamont selon lequel « la poésie doit être faite par tous non par un ». Les aspirations à l'égalité et à la démocratie très affirmées dans ces années-là facilitent à l'évidence l'expansion des ateliers d'écriture contre une vision élitiste et

(7) Tel Quel en fait la cible de son ironie. La question de l'influence de ces deux revues sur les ateliers d'écriture, de leurs points communs, de leurs conflits - largement idéologiques plus que proprement littéraires - justifierait un travail de thèse.

(8) Je renvoie pour une présentation détaillée de la contrainte oulipienne à l'article de C. Bisenius-Penin (2005).

(9) Il est à noter que le premier numéro de Change s'intitule «Le montage».

(10) La date de parution du livre ne doit pas masquer que la pratique des ateliers d'écriture par ces auteurs remonte aux années 1980 au sein du Groupe Français d’Éducation Nouvelle. 
patrimoniale de la littérature. Ceci est bien exprimé au début de Babel heureuse (1989) et explicité également dans les ateliers du GFEN. La dimension collective, opposée à l'individualité du génie, est commune aux revues d'avant-garde qui reflètent des positions de groupe et aux ateliers où sont volontiers pratiqués - plus ou moins selon les courants - la lecture des textes dans le groupe, l'écriture à plusieurs, l'échange de textes pour la réécriture ${ }^{(11)}$.

\subsection{Contre le primat de la signification, le travail du signifiant}

Quant au travail du texte, mis en exergue par les néo-romanciers, il revêt une importance capitale pour les ateliers d'écriture, dans la mesure où l'un de leurs enjeux est d'amener les scripteurs à améliorer leurs productions par un travail conscient de réécriture. Dans les ateliers de Claudette Oriol-Boyer (1980, 1990), ce retravail se fait par la recherche d'un équilibre entre signifié et signifiant qui manifeste combien la poétique de Jakobson imprègne les concepteurs d'ateliers. On retrouve cette même attention au pôle signifiant dans l'écriture effervescente théorisée par Ricardou (1978) et reprise par le GFEN ${ }^{(12)}$. Il s'agit dans cette consigne d'écriture de créer un stock de mots à partir d'un mot auquel on associe deux listes, l'une par la méthode classique des associations d'idées (axe idéel), l'autre par une recherche de mots présentant des sonorités communes, ou écrits avec les mêmes lettres (axe matériel). Or, c'est l'époque où sont publiés les cahiers d'anagrammes de Saussure (présentés dans Tel Quel $n^{\circ} 29$ et 37 par Kristeva, puis Starobinski). Saussure pensait que les poètes anciens s'étaient donné pour règle de faire réapparaître dans leurs textes sous forme anagrammatique le mot désignant le sujet central du poème. Il existe un lien direct entre cette hypothèse et l'emploi dans un texte des mots trouvés sur l'axe matériel : dans les deux cas, il s'agit de créer une cohérence du texte basée non plus sur les seuls signifiés mais aussi sur l'essaimage et le retour conscient des signifiants. Les consignes de réécriture mises en œuvre dans les ateliers ne prennent pas toutes appui sur le pôle signifiant du langage, mais sa mise en œuvre consciente apparaît clairement comme un antidote possible au primat de la signification et de la représentation. Élément essentiel de la fonction poétique, la paronomase (Jakobson 1963 : 219) est aussi un outil d'engendrement de la fiction : les animateurs d'ateliers s'inspirent alors de Raymond Roussel (1935), commençant une nouvelle par « la peau verdâtre de la prune un peu mûre » et la terminant par « la peau verdâtre de la brune un peu mûre».

Cette attention au signifiant s'explique aussi par la perméabilité des théoriciens et écrivains d'avant-garde à la psychanalyse et au rôle de l'inconscient dans les processus créatifs. Si Julia Kristeva, entrée au comité de Tel Quel en 1970, peut être considérée comme emblématique de cette tendance qui se réfère également à Jacques Derrida, Change, lui aussi, dans son " exploration collective du change des formes et du change matériel », fait place à une « plongée dans "l'inconscient des langues" et le “ça des mots" " $\left(\mathrm{n}^{\circ} 18,1974\right.$, p. 18). Pour Faye et ses collaborateurs, cela ne semble pas contradictoire avec la révérence pour Chomsky lequel, pourtant, comme le remarque à juste titre Tel Quel, construit sa théorie en faisant du locuteur un sujet cartésien universel et en excluant le signifiant « tel que la psychanalyse l'introduit de nos jours ", ainsi que " tout référent socio-historique » $\left(\right.$ Tel Queln ${ }^{\circ} 43,1970$, p. 79). La

(11) La question du rapport à l'autre dans l'atelier est au centre des réflexions d'Odette et Michel Neumayer ( $c f$. entre autres, $2003: 36-37,168-171$ et 2011 passim).

(12) On en trouvera une présentation détaillée dans Ricardou 1978 et un résumé synthétique dans Neumayer 2003: 43 . 
« fureur théorique » des années 60-70 s'accommode d'un certain éclectisme, et d'un aveuglement certain, si l'on pense aux éloges dithyrambiques de Tel Quel pour la révolution culturelle chinoise.

\section{4. À la recherche d'une théorie du sujet créateur}

La question du sujet, qui oppose parfois les revues, n'est pas non plus vraiment résolue par les animateurs d'ateliers d'écriture. Le soin d'Elisabeth Bing à faire renouer les participants avec leur enfance et leur désir enfoui d'écrire ${ }^{(13)} n$ 'est pas sans lien avec l'exploration psychanalytique. Dans les premiers temps de l'atelier, écritelle, « le passage par une écriture pulsionnelle est nécessaire», mais alterne avec une écriture de la «nomination la plus stricte du réel extérieur» (op. cit. p. 63) ${ }^{(14)}$. Dans son analyse du processus créateur, Alain André (1980) se réfère au travail du psychanalyste Didier Anzieu exposé dans Le Corps de l'œuvre. Dans l'ensemble, pourtant, les références à l'inconscient sont plus discrètes que dans les revues théoriques: dans leurs efforts pour légitimer les ateliers contre les accusations de thérapie sauvage, les animateurs ont à cœur de bien distinguer atelier et thérapie et insistent plus volontiers sur la mise à distance des émotions, sur le rôle du texte comme médiateur entre le sujet et ses affects. L'enjeu essentiel est, en effet, on l'a vu, de lutter contre une vision trop expressive de l'écriture. Par ailleurs, il est indéniable que la majorité des inventeurs d'ateliers à leurs débuts sont théoriquement, voire politiquement, très imprégnés par le structuralisme et le marxisme dominants qui ont entraîné ce qu'on a pu appeler la crise ou la mort du sujet.

Mais, d'un autre côté, leur pratique les confronte nécessairement à la question du sujet de l'écriture, de son désir et des sources de ses blocages ${ }^{(15)}$. Plusieurs s'efforcent d'aller au-delà de ces contradictions et mettent en lumière le paradoxe de tout acte de création, à la fois dessaisissement et transformation de celui qui s'y livre. Nicole Voltz dans Roche, Guiguet, Voltz (1989) propose au scripteur de passer d'une posture d'expression à une posture de créateur, dans laquelle le texte n'est pas là " pour exprimer [une] identité » mais pour exister indépendamment de celui qui l'écrit. Elle pense qu' « écrire c'est se retirer » ${ }^{(16)}$ mais elle dit aussi qu'écrire transforme l'identité de l'écrivain. Alain André affirme quant à lui «J'écris moins le texte que le texte ne m'écrit » (p. 10) et propose une troisième voie entre l'écriture vécue comme « expérience intérieure et entreprise d'exploration symbolique » et l'écriture centrée sur « l'objet textuel au mépris de l'auteur et du lecteur» (p. 14).

On voit ainsi apparaître des divergences sur la place et la conception du sujet aussi bien que sur le rôle attribué au signifiant, divergences dont témoignent les consignes des ateliers : certains ateliers se préoccupent peu de développer l'imaginaire de leurs scripteurs autrement que par la mise en œuvre de contraintes formelles ou par une transformation de textes littéraires disponibles, alors que d'autres varient les inducteurs, prenant appui tantôt sur des souvenirs d'enfance ou des mythes, tantôt sur des œuvres plastiques ou musicales, tantôt sur des fragments du réel. Il se dessine un

(13) Il s'agit en effet de produire des « récits multiples qui œuvrent à écarter les brouillards de la mémoire », 1992, p. 62.

(14) On retrouvera ce deuxième aspect dans les ateliers animés par François Bon (2000).

(15) On le voit bien dans l'article «Atelier d'écriture : une démarche de conquête du pouvoir d'écrire » GFEN, 1982, pp. 69-83, écrit par des militants pédagogiques aux convictions politiques très affirmées.

(16) Nicole Voltz reprend ici ce que dit Derrida de Jabès dans L'Écriture et la Différence, Seuil, 1967, p. 106. 
clivage entre ceux qui cherchent à équilibrer le pulsionnel et le symbolique en s'appuyant sur la réflexion psychanalytique et ceux qui cherchent dans les théories linguistiques un appui pour promouvoir la maîtrise des processus scripturaux. Le colloque de Cerisy de 1983 (dont les actes seront publiés par Oriol-Boyer en 1992) garde la trace de ces tensions, qui sont dues également à des phénomènes de " champ » au sens de Bourdieu : l'espace naissant des ateliers est marqué, comme tout espace public, par des luttes et rapports de force où chacun-e défend sa place en exacerbant parfois sa différence. En effet les divergences ne doivent pas occulter le fait que les inventeurs des ateliers partagent tous peu ou prou des convictions communes nées du climat intellectuel de l'époque. Certains « auteurs fétiches» se retrouvent d'ailleurs d'un courant à l'autre : Queneau, Perec, Calvino, nous l'avons vu, mais aussi les haïkus, Borges, Ponge, Sarraute (Enfance ou Tropismes), Leiris (Glossaire ou Biffures), et l'Aragon du « Mentir-vrai ». Le remplacement de "l'inspiration » par le travail, l'attention à la matérialité du texte et aux potentialités du signifiant, la mise en place de procédures permettant d'engendrer l'écriture à partir de contraintes matérielles, le rejet de la conception représentative du sens et l'appui sur le collectif sont, à des degrés divers, largement partagés et restent, me semble-t-il, déterminants dans les pratiques actuelles, même si l'évolution idéologique a parfois émoussé leur potentiel révolutionnaire.

\section{2. À l'orée du XXI ${ }^{\mathrm{e}}$ siècle : l'atelier d'écriture entre ouverture et récupération?}

On a vu que le riche terreau intellectuel des années 60 fournissait aux ateliers des armes théoriques vite transformées en outils d'animation et que, malgré des rivalités pour occuper un « champ » nouveau, les convergences l'emportaient largement sur les différences. Je voudrais maintenant envisager la situation présente des ateliers sous deux perspectives : celle de leurs interactions avec les évolutions du champ scolaire et universitaire en matière d'écriture, et celle de leurs éventuels rapports avec les théories qui analysent le discours et la production d'écrits.

\subsection{Le rapport texte/monde et l'écriture dans tous ses états}

On peut toujours opposer les ateliers qui partent du langage et de ses règles et ceux qui se proposent d'explorer par le langage un certain rapport au monde. Mais la plupart du temps les animateurs d'ateliers ont une vision dialectique des rapports texte/monde, à la manière de François Bon :

L'atelier d'écriture, si on met l'accent sur cette diction du monde plutôt que simplement sur ce qu'il est en fin de compte, production de langage, est toujours une prise de risque dans le rapport individuel au monde, et un déséquilibre dans ce risque ; celui qui conduit l'atelier, s'il fait prendre ce risque aux autres, doit pouvoir en énoncer les enjeux dans le seul univers du langage et ses formes, et que ce travail sur les formes du langage soit la barrière assez haute et forte pour que ce risque, côté participant, sinon s'annule, se transforme. (2000:10)

Sur le plan théorique, le courant anti-représentatif a perdu de sa force. Même s'il est difficile d'avoir une vue d'ensemble d'une production littéraire très éclatée, on ne peut que remarquer chez les romanciers actuels l'importance de l'autofiction, qui a acquis le statut de genre, de la biographie, fût-elle imaginaire ou réinventée, du rap- 
port à l'Histoire et à la mémoire familiale. Le Nouveau Roman continue à être étudié à l'Université mais la dimension réflexive et métatextuelle des œuvres des actuels écrivains de Minuit est beaucoup plus discrète et volontiers ironique ${ }^{(17)}$. Malgré l'existence de nombreuses et excellentes revues littéraires, on constate qu'elles n'ont guère l'ambition de fédérer tout un courant d'écrivains autour d'une position théorique forte ${ }^{(18)}$. Jean Ricardou et Daniel Bilous poursuivent l'entreprise de la textique mais n'ont pas fait tache d'huile. Ce sont les littératures à contraintes, dans le sillage de l'Oulipo dont on a célébré avec éclat le cinquantenaire ${ }^{(19)}$, qui constituent peut-être le projet collectif le plus dynamique et le plus susceptible de nourrir la réflexion des ateliers d'écriture ${ }^{(20)}$. L'échange entre Jean-Marie Laclavetine et Jean Lahougue publié en 1998 sous le titre Écriverons et liserons témoigne brillamment du conflit théorique et des arguments opposant les romanciers qui privilégient l'expérience personnelle et ceux qui accordent la primauté au dispositif et aux règles de production.

Mais le choix d'écrire sous contraintes n'exclut pas l'intérêt pour le monde tel qu'on peut le dire, comme en témoigne le numéro 14 de la revue Formules consacré aux « Formes urbaines de la création contemporaine ». Les évolutions rapides auxquelles nous sommes soumis sont une des urgences qui poussent à écrire et elles peuvent amener à construire des ateliers pour explorer ce monde en mutation à l'aide de formes nouvelles. Telle est la position de François Bon qui écrit par exemple à l'ouverture de Tous les mots sont adultes en 2000 :

Depuis dix ans, d'une façon quasi continue, les ateliers d'écriture ont été pour moi le lieu d'une découverte surprenante du monde lui-même, parce qu'en le nommant nous découvrons l'exigence pour l'écriture de s'ouvrir à des syntaxes et des formes neuves, que ce réel neuf exige, et qui nous le révèlent en retour. (p. 7)

Ses ateliers sont conçus comme des inventaires, des cartographies pour cadastrer nos villes et nos banlieues, recenser nos objets et nos propriétés, explorer notre identité fragmentaire et en constante métamorphose. S'appuyant sur un éventail d'auteurs très éclectique, la plupart résolument contemporains mais sans exclure Rabelais, Rimbaud ou Gracq, ils sont souvent bâtis sur des listes et font appel à la mémoire du passé récent, à la déambulation, au rêve. De tels exemples me semblent symptomatiques d'une sortie par le haut du conflit entre autoréférentialité et littérature représentative au profit d'une conception de l'écriture littéraire comme reconfiguration du monde.

Une autre piste s'est aussi ouverte avec le développement de la réflexion sur le rôle spécifique de l'écrit dans le champ des savoirs. Les travaux de Vygotski ou de Jack Goody, relayés par de nombreux auteurs ${ }^{(21)}$, ont montré combien l'écrit modifiait en profondeur les institutions et les situations d'apprentissage, et permettait par ailleurs de structurer les savoirs et d'acquérir une distance réflexive vis-à-vis du lan-

(17) Il s'est tenu en mai 2012 à Aix-en-Provence et Marseille un colloque organisé par le CIELAM intitulé « Existe-t-il un style Minuit?».

(18) On fera peut-être une exception pour la revue Nioques animée par Jean-Marie Gleize.

(19) 50 ans d'Oulipo. De la contrainte à l'oeuvre. C. Bisénius et A. Petitjean. PU Rennes 2012.

(20) Il a notamment donné naissance à la revue Formules.

(21) Pour une présentation et une discussion, on peut se référer à « A propos des usages de Goody en didactique » d’Yves Reuter, Pratiques n¹31-132, déc. 2006, pp. 131-154. 
gage. Il s'en est suivi notamment une utilisation des ateliers d'écriture à des fins autres que littéraires, comme outils permettant la conceptualisation, appuyée souvent sur un conflit socio-cognitif dont les étapes de l'atelier gardent les traces. Les livres d'Odette et Michel Neumayer, Animer un atelier d'écriture. Faire de l'écriture un bien partagé, publié en 2003, et Quinze ateliers pour une culture de paix, publié en 2010, offrent un bon exemple de cet élargissement du champ des ateliers d'écriture. Les auteurs ne séparent pas l'écriture comme pratique artistique de l'écriture comme instrument pour interroger notre rapport au monde, à la langue, à l'Histoire, d'où des références à des auteurs peu pratiqués dans les autres ateliers tels que Michaux, Kafka, Proust ou Simenon, mais aussi des ateliers sur Hiroshima, 1492, la guerre civile espagnole, l'Afrique aujourd'hui, les migrations, l'identité terrienne, la filiation ou l'altérité ${ }^{(22)}$. L'atelier permet alors de travailler sur les représentations de l'évènement ou de la notion, de les confronter entre elles, d'aborder le travail historique ou géographique par une écriture impliquée qui donne du sens à l'apprentissage. Par la mise en circulation de divers types d'écrits, l'atelier fait de la différence entre écriture intime et écriture théorique un objet d'analyse et permet de réfléchir aux normes régulant la production de ces différents écrits, aux fonctions qu'ils remplissent et aux procédures de validation dont ils font l'objet.

Mais ce recours aux ateliers d'écriture dans des champs professionnels variés et dans des disciplines autres que le français n'a pas encore fait l'objet de beaucoup d'analyses ${ }^{(23)}$. Il serait très important d'évaluer ce que la pratique de l'atelier d'écriture peut apporter dans ces domaines, de réfléchir aux dispositifs spécifiques à développer à cet effet, de ne pas confondre non plus le travail mené en ateliers d'écriture avec un simple brain-storming ou avec d'autres outils faisant appel à la créativité et à la pensée divergente. Il y aurait aussi à inventer des ateliers d'écriture dans le domaine de l'écriture de recherche, qui s'avère si difficile pour nombre d'étudiants. Les questions du travail du texte, du rapport à l'écriture, du rapport sujet / langage / monde, de la spécificité de la fiction, ou de celle de la production artistique pourront être envisagées autrement si l'on n'enferme pas les ateliers d'écriture dans le seul domaine littéraire.

\subsection{Atelier d'écriture ou écriture-imitation?}

L'animateur d'ateliers d'écriture ${ }^{(24)}$, qu'il soit oulipien ${ }^{(25)}$, binguien, alephien ou autre, sait que l'atelier, s'il est bien mené, permet l'émergence d'un sujet de l'écriture. Selon ses choix théoriques, il pensera différemment les rapports de ce sujet avec le sujet psychologique, avec la langue et les discours, avec le social ou l'inconscient, mais il sera attentif à ce que chacun puisse peu à peu construire une identité scripturale singulière. Oriol-Boyer (1990:16) insistait déjà sur le fait que le travail de relecture doit se pencher sur le respect de la consigne mais aussi sur la mise en évi-

(22) Le GFEN développe les ateliers d'écriture dans le champ philosophique comme on peut le voir dans l'ouvrage collectif Philosopher, tous capables publié par Chronique sociale en 2005.

(23) Au colloque de Cerisy de 2012, Emanuela Cocever a présenté un dispositif remarquable mis en place dans les parcours de formation des enseignants, travailleurs sociaux et soignants à Bologne. Cf. E. Cocever (a cura di) (2010), Scrittura e formazione, Erickson, Gardolo di Trento.

(24) Bien entendu, je me réfère ici à des animateurs/trices formé-e-s, et ayant la possibilité de réfléchir périodiquement avec d'autres sur leurs pratiques.

(25) $C f$. à ce sujet Wagner (2004). 
dence des « quelques règles implicites auxquelles [le scripteur] a obéi, à son insu, au moment de la rédaction » et elle assimile le scripteur en train de se relire à un «cryptanalyste » « essayant de découvrir le code qu'il a appliqué sans le savoir» (p. 35).

Il arrive souvent au début que ce code soit, non pas celui du scripteur, mais celui de genres ou de formes qu'il reproduit. L'émergence d'une langue à soi ne peut être que progressive, tissée d'emprunts à autrui, et l'atelier doit permettre de conscientiser au cours de son évolution tout ce que nous empruntons aux discours circulants : clichés, lieux communs, situations, «patrons ${ }^{(26)}$. Après la période structuraliste, les travaux de théorie littéraire des vingt dernières années ont bien montré, sous l'influence de Bakhtine et Volochinov et d'une réflexion approfondie sur l'intertextualité, combien l'appartenance des textes à des genres plus ou moins contraints guidait leur production et leur interprétation. Grâce à eux, nous disposons, plus encore qu'au début des ateliers d'écriture, d'un vaste réservoir de textes et de consignes qui permettent de travailler dans le sens de la parodie, de la transposition, du pastiche d'écrivain, de genre ou de registre ${ }^{(27)}$.

Par ailleurs, confrontés aux difficultés de langue et au bagage littéraire réduit de nombre d'étudiants, les animateurs d'ateliers en milieu scolaire ou universitaire ont tendance à multiplier les propositions bâties autour d'auteurs, généralement contemporains, sans qu'il soit question d'imitation au sens strict mais plutôt d'appropriation d'une problématique d'écriture ${ }^{(28)}$. L'atelier commence par un temps d'imprégnation où sont lus et analysés des extraits de romans ou des poèmes d'un même auteur, puis les participants sont invités à écrire dans le sillage de cet auteur. L'intention est louable : il s'agit de proposer des modèles différents de ceux diffusés par la culture de masse, de confronter les participants à des écrits souvent problématiques qui les orientent vers une posture plus réflexive. Mais dans les retours faits par l'animateur ${ }^{(29)}$, il arrive que les productions divergentes soient mal reçues, ce qui me semble aller à l'encontre de la construction d'un sujet de l'écriture singulier.

Le danger se trouve accru avec la confusion qui s'instaure parfois entre écriture d'invention et atelier d'écriture. L'écriture d'invention telle qu'elle a été mise en place au lycée entre 1999 et 2001 a fait une place, à côté des écrits métatextuels que sont la dissertation et le commentaire composé, aux écrits hypertextuels ${ }^{(30)}$. Reuter (2005) et Daunay (2005) ont bien analysé le processus de restriction qu'ont subi à la fois les modalités et les finalités de l'écriture d'invention au fil de son officialisation et des remous qu'elle a suscités :

Trois principes s'imposent pour encadrer l'écriture d'invention :

- la relation structurelles avec les textes littéraires, leur lecture et leur analyse :

l'écriture d'invention devient une actualisation de cette lecture-analyse ;

- l'équivalence entre écriture d'invention et réécriture-transposition de textes (par imitation, amplification, réduction...) ;

- le cadrage par des consignes explicites précisant genres, registres et situations d'énonciation et par des « exercices brefs et fréquents » (Reuter 2005 : 9).

(26) Sur cette notion, $c f$. Philippe (2008).

(27) Réécriture et écriture d'invention au lycée de Violaine Houdart-Mérot (Hachette 2004) fait très utilement le point sur ces notions et propose toute une panoplie d'exercices.

(28) Les actes à paraitre du colloque de Cergy de 2010, Les pratiques d'écriture littéraire à l'Université, Mongenot C. \& Houdart-Mérot V. (dir.), Champion, en fournissent plusieurs exemples.

(29) Sur les « retours » de l'animateur, voir Monte et Robet à paraitre.

(30) Je reprends à la suite de Daunay $(2005: 25)$ la distinction de Genette 2002. 
Les programmes de l'Épreuve Anticipée de Français définis par la note de service 2006-199 du 4/12/2006 voient dans l'écriture d'invention un moyen de vérifier la qualité de la lecture des textes du corpus proposé et accordent le primat à une écriture à visée argumentative souvent métatextuelle ( $c f$. Daunay : 26-27) ou à des récits hypertextuels :

L'exercice se fonde, comme les deux autres, sur une lecture intelligente et sensible du corpus, et exige du candidat qu'il se soit approprié la spécificité des textes dont il dispose (langue, style, pensée), afin d'être capable de les reproduire, de les prolonger, de s'en démarquer ou de les critiquer. Le document iconographique, s'il est joint au corpus, ne peut pas servir de support. En aucun cas, il ne sera demandé d'en faire une étude pour lui-même. Comme elle doit se prêter à une évaluation objective des correcteurs, l'écriture d'invention doit se fonder sur des consignes claires et explicites. Elle s'inscrit dans le programme défini par les objets d'étude de la classe de première.

Elle peut prendre des formes variées. Elle peut s'exercer dans un cadre argumentatif :

- article (éditorial, article polémique, article critique, droit de réponse...) ;

- lettre (correspondance avec un destinataire défini dans le libellé du sujet, lettre destinée au courrier des lecteurs, lettre ouverte, lettre fictive d'un des personnages présents dans un des textes du corpus, etc.);

- monologue délibératif ; dialogue (y compris théâtral) ; discours devant une assemblée ;

- récit à visée argumentative (fable, apologue...).

Mais, lorsqu'elle concerne le genre narratif, elle peut s'appuyer sur des consignes impliquant les transformations suivantes :

- des transpositions : changements de genre, de registre, ou de point de vue ;

- ou des amplifications : insertion d'une description ou d'un dialogue dans un récit, poursuite d'un texte, développement d'une ellipse narrative...

Ces infléchissements curriculaires n'ont que peu d'influence sur les ateliers d'écriture à visée de développement personnel. En revanche, ils pèsent de tout leur poids sur les pratiques d'écriture dans un cadre scolaire, au collège comme au lycée. Ils risquent aussi d'influencer les ateliers d'écriture qui se développent - et on ne peut que s'en réjouir - dans les cursus de lettres à l'Université ${ }^{(31)}$. Or cette conception de l'écriture d'invention va nettement dans le sens d'une pratique du pastiche et de la parodie. Comme je l'ai dit plus haut, le travail de transposition et d'imitation est intéressant pour permettre aux scripteurs de prendre conscience de la part prédominante des discours autres dans ce que nous croyons être notre propre discours. En la rendant explicite, en en faisant un moteur d'écriture, il affranchit de l'obsession d'originalité et de spontanéité qui est souvent très présente au début chez les participants aux ateliers d'écriture. Mais il devrait à mon sens n'occuper qu'une place limitée dans les différentes séances prévues par l'animateur, au début, par exemple, comme tremplin, ou en cours de route, ponctuellement, quand la question des stéréotypes à dépasser s'avère cruciale. Si, dans les années 60 , la mise en avant de l'intertextualité a été extrêmement salutaire pour aider à écrire, sa généralisation-que l'on observe aussi dans la littérature publiée - me semble présenter le risque d'un retour à

(31) Pour avoir une idée de ce développement, on peut se reporter aux informations collectées par le réseau « pratiques d'écriture à l'Université » et mises en ligne par le Centre de Recherches Textes et Francophonies de l'Université de Cergy-Pontoise : http://www.u-cergy.fr/fr/laboratoires/labo-crtf/reseau-pratiques-d-ecriture-a-l-universite.html 
la conception autoréférentielle de la littérature au détriment de sa capacité à reconfigurer le monde, telle que je l'évoquais plus haut.

Je préfère, quant à moi, défendre une certaine conception des ateliers d'écriture qui résiste à une trop grande accommodation aux injonctions institutionnelles et aux pratiques lettrées et qui se démarque nettement de l'écriture d'invention. Je me situerais plutôt dans la lignée de Freinet qui voulait donner à l'enfant le pouvoir de parler en son nom propre, je dirais pour ma part le pouvoir de choisir sa distance et de construire sa propre identité langagière ${ }^{(32)}$. La fréquentation des textes littéraires me semble indispensable dans les ateliers d'écriture à visée esthétique mais leur place demande à être soigneusement pensée et plutôt que de centrer la séance sur une seule écriture, il est préférable, bien souvent, de proposer plusieurs façons de résoudre la problématique d'écriture que l'on souhaite travailler. Je ne voudrais d'ailleurs pas défendre un type d'atelier contre un autre, mais plutôt mettre l'accent sur une clarification nécessaire entre ce qu'on peut à bon droit appeler « atelier d'écriture » et ce qu'il vaudrait mieux nommer « dispositif d'entrainement à l'écriture créative ». Il ne faut pas oublier en effet que les ateliers d'écriture ont été inventés contre l'institution scolaire, et qu'une grande part de leur efficace tient dans cette position qui se manifeste à trois niveaux :

- le dispositif : le maître surplombant est remplacé par un animateur qui accompagne une recherche individuelle et collective dont il perd au moins en partie la maitrise ;

- les fondements théoriques : l'écriture est un travail sur le langage faisant l'objet d'une analyse réflexive ;

- les objectifs : le scripteur passe du statut de lecteur à celui de producteur de textes et se construit en tant que sujet dans ce processus à finalité explicitement citoyenne et émancipatrice.

L'oubli de l'une ou l'autre de ces dimensions conduit à une dénaturation de l'atelier d'écriture : on reconduit la coupure expert / novice au détriment de la recherche collective, on oublie la dimension réflexive dans une fuite en avant dans le faire, on vise à faire de bons techniciens plus que des personnes confrontées à des problématiques de création.

Le travail autour et avec les auteurs est un bon poste d'observation de ces éventuelles dérives. Si une phase d'imitation n'est pas forcément à proscrire, l'atelier ne doit pas s'y réduire, ni commencer, à mon avis, par cela. Imaginons que l'on veuille travailler sur le rythme : celui-ci doit d'abord être envisagé dans toute l'étendue problématique du concept ${ }^{(33)}$ par une ouverture - ou des inducteurs - qui confronte[nt] rythmes naturels, humains, artistiques et amène[nt] à une première écriture. On peut ensuite proposer un éventail de textes littéraires qui travaillent le rythme chacun à sa façon et dans lesquels les scripteurs pourront puiser des solutions aux problèmes qu'ils ont d'abord rencontrés eux-mêmes. On pourra confronter la longue phrase sinueuse à la Claude Simon ou à la Proust - fort différentes l'une de l'autre, d'ailleurs et la phrase lapidaire à la Duras ou la Ernaux, on pourra s'essayer provisoirement à l'une ou l'autre, mais ceci viendra en prolongement d'un obstacle sur lequel on a achoppé, d'une question que l'on s'est posée et qui auront été nommés. Ce qui sera en

(32) Pour une confrontation entre l'écriture d'invention et le texte libre, on pourra lire Bishop 2005.

(33) Je renvoie à Critique du rythme, Henri Meschonnic, Lagrasse, éd. Verdier 1982 et à Rythme et sens. Des processus rythmiques en poésie contemporaine, Lucie Bourassa, Montréal, éd. Balzac, 1993. 
jeu, ce sera la façon dont un sujet se débat avec un matériau, la linéarité de la langue, ses accents, ses pauses, dans un processus qui part d'une émotion, d'une représentation, ou d'une hypothèse, et qui cherche à la transformer en langage. Ce faisant, le scripteur aura fait des choix qui, analysés en atelier, confrontés à d'autres, lui apprendront des choses sur son rapport à la langue et au monde. Souvent les animateurs d'ateliers préfèrent partir d'un texte d'auteur parce qu'ils craignent d'enfermer les scripteurs dans leurs ressources propres, parfois limitées par des lectures trop réduites, et formatées par des stéréotypes et des clichés. Mais ce faisant, ils empêchent la phase nécessaire de tâtonnement qui suscitera ensuite la lecture des auteurs à la recherche de réponses possibles. Plus qu'avec l'œuvre finie, l'atelier devrait permettre la rencontre avec le travail du créateur, avec ses questionnements. Au lieu de partir d'une page de La Rage de l'expression ${ }^{(34)}$ sur l'œillet, le mimosa ou le bois de pins, il vaut mieux proposer au scripteur une tâche de description-définition d'un objet naturel ou fabriqué qu'il aura choisi, lui faire consulter les dictionnaires, rechercher des mots autour de son objet par la méthode de l'écriture effervescente, et seulement ensuite s' appuyer sur les écrits pongiens lors de la phase réflexive de l'atelier et de la réécriture qui la suit.

Ce que l'atelier d'écriture met en question, c'est le rapport scolaire ordinaire à la connaissance. Il relève en effet de la construction d'une situation-problème (Petitjean 2005 : 77) mais il n'aboutit que partiellement, à la différence d'autres situations-problèmes, à un savoir stabilisé, et celui-ci est de type procédural et non déclaratif. Contrairement à l'écriture d'invention telle qu'elle se pratique en vue de l'E.A.F., l'atelier reste très ouvert quant à ses effets à court terme, qui seront très différents d'un participant à l'autre, porteront tantôt sur le rapport à soi, tantôt sur les représentations du monde ou de la langue, et ne seront mesurables qu'en faisant parler le scripteur de sa pratique, en l'analysant avec lui. Ce qui est peut-être difficile pour des animateurs issus du monde scolaire, c'est d'accepter que les savoirs construits en atelier ne soient pas homogènes, que chacun avance à son rythme, découvre des choses différentes de son voisin ${ }^{(35)}$, et qu'une grande partie de ce savoir opératoire reste implicite. L'idéal scolaire du savoir décontextualisé et réutilisable reste ici inopérant. Cela conditionne aussi un rapport aux auteurs très spécifique qui se distingue du rapport usuel, comme le soulignent Odette et Michel Neumayer :

Les auteurs nous ouvrent au monde de l'écriture pour peu que nous décidions d'y entrer. La référence à leur œuvre, à ce qui pour eux a souvent été l'activité centrale de toute une vie, est une des façons d'ancrer le travail qui se fait dans le cadre de l'atelier à un hors-cadre lié à l'histoire littéraire, au patrimoine culturel et social.

La présence/absence de l'auteur ouvre la possibilité aux sujets écrivants de prendre du recul par rapport à leur désir (ou leur crainte) d'écrire. Elle triangule leur relation avec les animateurs. [...]

Mais la référence aux auteurs est aussi une source potentielle de malentendus. [...] Un atelier n'est pas une leçon qui serait simplement plus "active", avec de beaux exercices amusants, surprenants, innovants. [...] L'auteur est celui qui a su faire preuve d'audace et qui peut nous inciter à être audacieux à notre façon, à notre tour. $(2003: 55-56)$

(34) Francis Ponge, coll. Poésie / Gallimard, première publication Mermod, Lausanne, 1952.

(35) En réalité, c'est ce qui se passe aussi pour d'autres formes de savoir dans d'autres dispositifs mais l'évaluation sommative fait l'impasse sur ces différences et promeut l'idée fausse d'un savoir homogène et complètement détaché des conditions d'apprentissage du sujet. 
La dimension réflexive des ateliers, si importante, ne doit pas occulter le fait que les savoirs qu'elle fait émerger sont radicalement différents des savoirs déclaratifs et restent constamment ancrés dans une pratique et une subjectivité.

\subsection{Nouveaux paradigmes linguistiques et interactions possibles en- tre chercheurs et animateurs d'ateliers d'écriture}

Les ateliers d'écriture se sont développés de façon autonome depuis les années 1980 et ont produit leurs propres théories, ce qui est heureux. Mais il semblerait que le contact ait été quelque peu distendu avec les chercheurs qui étudient le langage et les textes. Je voudrais dans cette dernière partie indiquer quelques pistes pour une collaboration entre animateurs d'ateliers, théoriciens de la littérature ou linguistes et didacticiens de l'écriture. Cela me permettra d'indiquer à grands traits quelques modifications notables dans le paradigme épistémologique depuis les années 60 et de préciser quels savoirs récents peuvent à présent irriguer les ateliers d'écriture, comme la narratologie et les travaux sur l'intertextualité ont pu nourrir leurs débuts.

Le premier domaine de savoir est celui de la critique génétique. Celle-ci nous a permis de mieux comprendre comment s'élaborent les textes littéraires et elle fournit des outils précieux pour organiser la phase de réécriture des ateliers, notamment lorsque ceux-ci s'engagent sur des projets longs, exigeant une planification. L'ouvrage collectif Critique génétique et didactique de la réécriture dirigé par OriolBoyer (2003) montre tout ce que l'exploitation des avant-textes peut faire gagner à une didactique de l'écriture en situation scolaire mais peut aussi inspirer des consignes de réécriture en atelier. Cette approche peut être complétée par les travaux des cognitivistes sur la réalisation des tâches d'écriture et ceux des linguistes sur la textualité ${ }^{(36)}$. Par rapport au début des ateliers d'écriture, on dispose à présent de connaissances beaucoup plus précises sur les différents éléments qui fondent la cohérence des textes, sur leur processus d'élaboration et de révision, y compris par des observations en temps réel de tâches réalisées sur ordinateur.

Le développement de la linguistique énonciative peut, quant à lui, nourrir la réflexion des animateurs sur la question du sujet de l'écriture, mais surtout pourrait inciter les chercheurs à travailler davantage sur les corpus que constituent les textes produits en atelier. On sait que le paradigme dominant en linguistique a complètement changé depuis la naissance des ateliers. On est passé du structuralisme centré sur le texte et de la conception abstraite du sujet parlant dans la théorie chomskyenne, au dialogisme bakhtinien, aux théories polyphoniques de l'énonciation, à l'interactionnisme et à la prise en compte des variations langagières. La plupart des théories linguistiques ont intégré ces données et, même si des descriptions du système de la langue demeurent nécessaires, la majorité des travaux prend en compte les évolutions historiques, les réorganisations continues du système, l'émergence de nouvelles formes. La conception du sujet s'est considérablement enrichie en distinguant le sujet parlant et les voix et discours qui le traversent, ou dont il organise le dialogue, selon qu'on est plutôt, pour reprendre les termes de Jacqueline

(36) Pour une initiation à ces travaux, on peut consulter Piolat, A. \& Pélissier, A. (éds), La rédaction de textes. Approche cognitive, Delachaux et Niestlé, 1998 ; Fayol, M. Des idées au texte. Psychologie cognitive de la production verbale, orale et écrite, PUF, 1998 et Adam, J.-M., La linguistique textuelle, Armand Colin, 2011. 
Authier-Revuz ${ }^{(37)}$, du côté de l'hétérogénéité constitutive ou de l'hétérogénéité montrée. La construction interactive de l'objet du discours dans la conversation, les négociations sur les choix de nomination et sur la place des locuteurs, les phénomènes d'anticipation et de reprise au sein d'un discours apparemment monologique sont maintenant beaucoup mieux étudiés. On peut donc envisager que se développent les études sur des corpus de textes produits et retravaillés en atelier d'écriture afin de mieux comprendre comment le sujet de l'énonciation, distinct du sujet empirique ${ }^{(38)}$, émerge et s'affirme dans une « campagne» de textes. Ce travail a été initié par D. Bucheton (1995), poursuivi par J. Lafont-Terranova (2009) et me semble une des pistes les plus prometteuses pour une collaboration entre animateurs d'ateliers d'écriture et linguistes. L'observation de phénomènes tels que les discours rapportés, les choix de points de vue, les gloses métadiscursives conduirait à préciser le positionnement du locuteur, et l'influence éventuelle des consignes ou de l'image de soi sur ce positionnement.

On aura intérêt également à tenir compte du regard d'analystes du discours tels que Dominique Maingueneau (2004) qui pense la littérature comme pratique sociale cherchant constamment à s'auto-instituer et dont les réflexions sur l'éthos et la scénographie permettent de relier les questions énonciatives à des enjeux sociopragmatiques. Permettre aux participants de se construire comme sujets dans l'écriture exige en effet d'être très conscient de ce qui se passe lors de l'élaboration d'écrits littéraires : les modèles existants en matière de littérature et d'écrivains, les consignes de l'animateur, l'image que se fait le scripteur de son activité et de sa place au sein du groupe entrent en interaction de façon complexe. Une tentation toujours latente consiste à vouloir réduire cette complexité en donnant une place démesurée à la technique ou à l'imitation au détriment des enjeux éthiques et artistiques, ou inversement à penser qu'une bonne compréhension des enjeux va permettre l'émergence miraculeuse d'une langue à soi chez chaque participant sans qu' on en passe par une confrontation lucide aux formes reconnues et aux normes établies.

La multiplication des connaissances sur l'écriture, sur le discours littéraire, sur la subjectivité énonciative, fait surgir toutefois deux problèmes. Tout d'abord, il faut se demander comment ces connaissances peuvent être transmises dans le cadre des formations à l'animation d'ateliers d'écriture. Mais surtout l'animateur un peu au fait de ces nouveaux savoirs risque de trop vouloir planifier et décomposer le processus d'écriture, dans l'intention louable de faciliter la tâche aux scripteurs, mais au détriment de la dialectique entre maîtrise et non-maîtrise essentielle à toute création artistique. S'il est souhaitable que l'animateur sache le plus de choses possibles sur l'écriture, il doit se garder de se transformer en professeur d'écriture. L'atelier, contrairement à ce qui se passe d'ordinaire dans l'espace scolaire, est un lieu où l'apprentissage privilégie le faire et la confrontation entre pairs plutôt que le dire et la relation maître/élève. C'est un lieu où la temporalité oscille entre le temps bref de la prise de conscience initiale, du bouleversement par rapport à ce qu'on croyait savoir ou ne pas savoir, temps de rupture à ménager périodiquement dès que trop de certitudes s'installent, et le temps long de la découverte par soi-même, avec l'aide du groupe, de ses potentialités et de ses désirs ainsi que des outils nécessaires pour aller plus loin. Vouloir hâter cette découverte, améliorer rapidement les textes, les faire correspondre aux attentes de l'animateur, c'est empêcher une véritable appropriation par le scripteur des outils proposés, c'est en rester à une perspective de transmis-

(37) Ces mots qui ne vont pas de soi, Larousse, 1995.

(38) On pourra lire sur cette question les rappels théoriques contenus dans Boré (2011). 
sion plus que d'auto-socio-construction, pour reprendre le terme de Wallon diffusé par le GFEN. Choisir l'atelier d'écriture, c'est choisir la lenteur, mais une lenteur active où chaque étape construit celui qui l'entreprend et lui permet de progresser.

\section{Conclusion}

Le lien entre les ateliers d'écriture et les théories littéraires et linguistiques est très fort lors de la naissance des ateliers mais plutôt unilatéral : les théories fournissent aux animateurs d'ateliers des armes pour justifier leur pratique encore nouvelle et des outils concrets pour imaginer des ateliers qui cassent l'illusion représentative et la primauté accordée à la signification afin de mettre l'écriture à la portée de tous. Au fil du temps, les ateliers évoluent selon leur propre logique, développant par exemple l'écriture à partir de textes d'auteurs ou l'exploration de situations historiques ou de concepts philosophiques. Ils sont également perméables aux changements institutionnels réintroduisant l'écriture d'invention au lycée de sorte que se produit parfois un certain effacement des frontières entre ateliers d'écriture et entraînement à l'écriture d'invention. L'accroissement considérable de nos connaissances sur les processus d'écriture et sur le dialogisme sous-jacent à toute production verbale devrait enrichir les ateliers d'écriture, notamment pour la mise en œuvre de la réécriture et pour une meilleure prise en compte des processus de construction du sujet scripteur en interaction avec autrui. Il faudrait pour cela que les chercheurs s'intéressent davantage aux corpus produits en ateliers d'écriture et que les animateurs d'ateliers restent toujours friands du lien théorie/pratique qui a fait la force des ateliers à leurs débuts. De nombreuses recherches sont à mener sur des questions aussi cruciales pour les ateliers que l'interaction entre soi et autrui, la triangulation sujet /langue/monde et le lien étroit entre contraintes et stimulation de l'imaginaire. Cependant la visée émancipatrice de l'atelier d'écriture doit rester première contre tout risque de didactisation, ce qui passe par une posture d'animation spécifique qui fasse la part belle aux questionnements des scripteurs et laisse une part prépondérante à leur propre analyse réflexive. 


\section{Références}

ANDRÉ, A. (1989) : Babel heureuse : l'atelier d'écriture au service de la création littéraire, Paris, Syros.

ANZIEU, D. (1981) : Le corps de l'œuvre, Paris, Gallimard.

BILOUS, D. (2004) : «Sur la mimécriture. Essai de typologie », dans Du pastiche, de la parodie et de quelques notions connexes, dir. Paul Aron, Québec, Éditions Nota Bene, pp. 103-136.

— (2009) : «La mimécriture : règles d'un art», Modèles linguistiques, tome XXXVI, vol.60, pp. 29-53.

BISENIUS-PENIN, C. (2005) : «Écriture d'invention au lycée et écriture à contraintes », Pratiques $\mathrm{n}^{\circ} 127 / 128$, pp. 209-229.

BING, E. (1976) : Et je nageai jusqu'à la page, éd. des Femmes.

- (1992) : «Histoire d'une pratique, ses postures, ses risques » dans OriolBoyer (dir.) pp. 59-72.

Bishop, M.-F. (2005) : «Texte libre et écriture d'invention, quel rapport? », Pratiques $\mathrm{n}^{\circ} 127 / 128$, pp. 143-153.

BON, F. (2000) : Tous les mots sont adultes, Paris, Fayard.

BONIFACE, C. (avec la collab. d'O. PIMET) (1992): Les ateliers d'écriture, Retz.

BORÉ, C. (2011) : «L'énonciation des brouillons et la question du sujet scolaire», Pratiques $\mathrm{n}^{\circ}$ 149/150, pp. 71-90.

Bucheton, D. (1995) : Écritures, réécritures, récits d'adolescents, Berne, Peter Lang.

DAUNAY, B. (2005) : «Les ambigüités des textes officiels sur l'écriture d'invention », Pratiques $n^{\circ} 127 / 128$, pp. 17-30.

Genette, G. (1982) : Palimpsestes, Paris, Seuil.

GFEN (H. Bassis dir.) (1982) : Quelles pratiques pour une autre école?, Casterman.

HOUdART-MÉROT, V. (2004) : Réécriture et écriture d'invention au lycée, Hachette.

JAKOBSON, R. (1963) : Essais de linguistique générale, tome 1, trad. N. Ruwet, Paris, Minuit.

KRISTEVA, J. (1969) : Semeiotikê. Recherches pour une sémanalyse, Paris, Seuil.

LaClavetine, J.-M. et Lahougue, J. (1998) : Écriverons et liserons, Seyssel, Champ Vallon.

LAFONT-TERRANOVA, J. (2009) : Se construire à l'école comme sujet-écrivant : l'apport des ateliers d'écriture, Diptyque $\mathrm{n}^{\circ} 15$, Presses Universitaires de Namur.

Maingueneau, D. (2004) : Le discours littéraire. Paratopie et scène d'énonciation, Armand Colin.

MONTE, M. et RoBet, C. (à paraître) : «Les retours écrits de l'animateur sur les textes produits en atelier : principes et effets », in Mongenot, C. \& HoudartMérot, V., Actes du colloque Les pratiques d'écriture littéraire à l'Université, Champion. 
NEUMAYER, O. et M. (2003) : Animer un atelier d'écriture. Faire de l'écriture un bien partagé, ESF.

- (2010): Quinze ateliers pour une culture de paix, Éd. Chronique sociale.

ORIOL-BOYER, C. (1980) : «Lire pour écrire. Atelier d'écriture et formation des maîtres », Pratiques n ${ }^{\circ}$ 26, pp. 94-112.

- (dir.) (1990) : La Réécriture, Actes de l'Université d'été de Cerisy-laSalle de 1988, Éd. L'Atelier du texte.

- (dir.) (1992) : Ateliers d'écriture (1992), Actes de l'Université d'été de Cerisy-la-Salle de 1983, Éd. L'Atelier du texte.

- (dir.) (2003) : Critique génétique et didactique de la réécriture, Éd. Bertrand-Lacoste.

Petitjean, A. (1984a) : «Pastiche et parodie, enjeux théoriques et pédagogiques ", Pratiques n ${ }^{\circ} 42$, pp. 3-33.

— (1984b) : «"Le petit chaperon rouge" : contes et anticontes », Pratiques $\mathrm{n}^{\circ} 42, \mathrm{pp} .63-78$.

- (2005) «Écriture d'invention au lycée et acquisition de savoir et de savoir faire », Pratiques $\mathrm{n}^{\circ} 127 / 128$, pp. 75-96.

Philippe, G. (2008) : «Registres, appareils formels et patrons », dans GaudinBordes, L. \& Salvan, G., Les registres. Enjeux stylistiques et visées pragmatiques, Louvain, Academia-Bruylant, pp. 27-37.

REUTER, Y. (2005) «L'écriture d'invention : réflexions didactiques sur une réforme en cours », Pratiques $n^{\circ} 127 / 128$, pp. 7-16.

Revue CHANGE (1968-1983), Le Seuil puis Seghers.

Revue FORMULES, Presses Universitaires du Nouveau Monde, site Internet : http ://www.ieeff.org/formulessitenewhome.html

Revue TEL QUEL (1960-1982), Le Seuil.

Ricardou, J. (1978) : «Écrire en classe » Pratiques n² 20, pp. 23-70.

Roche, A., Guiguet, A., Voltz, N. (1989 ${ }^{1}$ Bordas) : L'atelier d'écriture : éléments pour la rédaction du texte littéraire, 2005 rééd. Armand Colin.

Rossignol, I. (1996) : L'Invention des ateliers d'écriture en France, L'Harmat$\tan$.

Roussel, R. (1935) : Comment j'ai écrit certains de mes livres, A. Lemerre.

WAGNER, F. (2004) : "Le sujet sous contrainte(s) » in Blankeman, B., MuraBrunel, A., et Dambre, M. (dir.) Le roman français au tournant du XXI siècle, Presses de la Sorbonne nouvelle, pp. 431-438.

WECK, F. (2007) : Faire écrire étudiants et lycéens, L'Harmattan. 